\title{
De Novo Synthesized Estradiol Protects against Methylmercury-Induced Neurotoxicity in Cultured Rat Hippocampal Slices
}

\author{
Takeshi Yamazaki $^{1 * 9}$, Megumi Yamamoto ${ }^{29}$, Yasuhiro Ishihara ${ }^{1}$, Shota Komatsu ${ }^{1}$, Eiji Munetsuna ${ }^{10}$, \\ Masahiro Onizaki ${ }^{1}$, Atsuhiko Ishida ${ }^{1}$, Suguru Kawato ${ }^{3}$, Takao Mukuda ${ }^{4}$
}

1 Laboratory of Molecular Brain Science, Graduate School of Integrated Arts and Sciences, Hiroshima University, Higashi-Hiroshima, Japan, 2 Physiology Section, Department of Basic Medical Sciences, National Institute for Minamata Disease, Minamata, Kumamoto, Japan, 3 Department of Biophysics and Life Sciences, Graduate School of Arts and Sciences, The University of Tokyo at Komaba, Meguro, Tokyo, Japan, $\mathbf{4}$ Laboratory of Integrative Physiology, Graduate School of Integrated Arts and Sciences, Hiroshima University, Higashi-Hiroshima, Japan

\begin{abstract}
Background: Estrogen, a class of female sex steroids, is neuroprotective. Estrogen is synthesized in specific areas of the brain. There is a possibility that the de novo synthesized estrogen exerts protective effect in brain, although direct evidence for the neuroprotective function of brain-synthesized estrogen has not been clearly demonstrated. Methylmercury (MeHg) is a neurotoxin that induces neuronal degeneration in the central nervous system. The neurotoxicity of MeHg is regionspecific, and the molecular mechanisms for the selective neurotoxicity are not well defined. In this study, the protective effect of de novo synthesized $17 \beta$-estradiol on $\mathrm{MeHg}$-induced neurotoxicity in rat hippocampus was examined.

Methodology/Principal Findings: Neurotoxic effect of MeHg on hippocampal organotypic slice culture was quantified by propidium iodide fluorescence imaging. Twenty-four-hour treatment of the slices with $\mathrm{MeHg}$ caused cell death in a dosedependent manner. The toxicity of MeHg was attenuated by pre-treatment with exogenously added estradiol. The slices de novo synthesized estradiol. The estradiol synthesis was not affected by treatment with $1 \mu \mathrm{M} \mathrm{MeHg}$. The toxicity of MeHg was enhanced by inhibition of de novo estradiol synthesis, and the enhancement of toxicity was recovered by the addition of exogenous estradiol. The neuroprotective effect of estradiol was inhibited by an estrogen receptor (ER) antagonist, and mimicked by pre-treatment of the slices with agonists for $E R \alpha$ and $E R \beta$, indicating the neuroprotective effect was mediated by ERs.
\end{abstract}

Conclusions/Significance: Hippocampus de novo synthesized estradiol protected hippocampal cells from MeHg-induced neurotoxicity via ER $\alpha$ - and ER $\beta$-mediated pathways. The self-protective function of de novo synthesized estradiol might be one of the possible mechanisms for the selective sensitivity of the brain to MeHg toxicity.

Citation: Yamazaki T, Yamamoto M, Ishihara Y, Komatsu S, Munetsuna E, et al. (2013) De Novo Synthesized Estradiol Protects against Methylmercury-Induced Neurotoxicity in Cultured Rat Hippocampal Slices. PLoS ONE 8(2): e55559. doi:10.1371/journal.pone.0055559

Editor: Allan Siegel, University of Medicine \& Dentistry of NJ - New Jersey Medical School, United States of America

Received September 25, 2012; Accepted December 27, 2012; Published February 6, 2013

Copyright: (c) 2013 Yamazaki et al. This is an open-access article distributed under the terms of the Creative Commons Attribution License, which permits unrestricted use, distribution, and reproduction in any medium, provided the original author and source are credited.

Funding: This work was supported by a Grant-in-Aid for Scientific Research from the Ministry of Education, Culture, Sports, Science and Technology of Japan. The funders had no role in study design, data collection and analysis, decision to publish, or preparation of the manuscript.

Competing Interests: The authors have declared that no competing interests exist.

*E-mail: takey@hiroshima-u.ac.jp

9 These authors contributed equally to this work.

a Current address: Department of Biochemistry, School of Medicine, Fujita Health University, Toyoake, Aichi, Japan

\section{Introduction}

Estrogen, a type of female sex steroid, is an important factor in the brain, where it has non-reproductive functions such as memory enhancement, neurotrophic action, and neuroprotection, as well as a role in reproductive behavior [1]. A protective effect of estrogen in Alzheimer's disease, Parkinson's disease and acute ischemic stroke has been demonstrated [2,3,4]. Estrogen is also neuroprotective against many insults, such as serum or oxygenglucose deprivation, amyloid $\beta$ peptide-induced toxicity, glutamate-induced excitotoxicity, and the toxicities of various chemicals, including $\mathrm{MeHg}[5,6,7,8,9,10,11]$.
Brain estrogen can be derived from peripheral steroidogenic organs via the blood stream, and from de novo synthesis at specific brain regions from steroid precursors or cholesterol [12]. Neurosteroids are synthesized from cholesterol in the central and peripheral nervous systems through mechanisms that are independent of peripheral steroidogenic organs, which include gonads and adrenal glands [12]. Estradiol concentrations were the same or higher in the newborn rat brain after removing the gonads and adrenals compared with sham-operated rats, indicating contribution of de novo estradiol synthesis as a neurosteroid [13]. The hippocampus is one of the regions that actively synthesize estradiol. The rat hippocampal cultured slices and dispersed cells synthesize $17 \beta$-estradiol as a neurosteroid $[14,15,16]$. In adult rats, 
the estradiol content of the hippocampus was 6 times higher than in plasma, indicating de novo synthesis of estradiol there [17].

Methylmercury $(\mathrm{MeHg})$ is a hazardous pollutant and humans are exposed to $\mathrm{MeHg}$ mainly through consumption of fishes $[18,19]$. Minamata disease, anthropogenic exposure to $\mathrm{MeHg}$ in Japan, and the $\mathrm{MeHg}$ poisoning in Iraq have established the toxicity of $\mathrm{MeHg}$ in the nervous system [20,21,22]. MeHg enters the central nervous system through the blood-brain barrier, and deposits of inorganic mercury are present diffusely in the brain. The lesions of Minamata disease are, however, localized in specific regions of the brain. Some parts of the cerebral and cerebellar cortices are affected more severely than other parts. There is no significant correlation between mercury deposition and lesion distribution [23,24]. Molecular mechanisms for the selective susceptibility of each area of the brain to $\mathrm{MeHg}$ are not well defined, and they must be analyzed in order to understand the clinical symptoms [24].

The toxic effect of $\mathrm{MeHg}$ has been shown to be attenuated by exogenous estradiol in primary cultured rat granule cells [11]. In male mice, the administration of estradiol partially prevented motor activity deficits and the modification of cerebellar glutathione metabolism induced by $\mathrm{MeHg}$ [25]. Estrogen protects the brain through multiple mechanisms, both dependent on and independent of estrogen receptor (ER) activity. In the cultured granule cells, estradiol protected against $\mathrm{MeHg}$ toxicity by acting as an antioxidant. In these cells, estradiol functions the same way as J811, which is an artificial antioxidant that protects the cells without stimulation of estrogen receptors [11]. On the other hand, ER-mediated neuroprotection against excitotoxic glutamate and global ischemia has been shown to prevent neurodegeneration in cultured rat hippocampal neurons [26]. In the primary cultured neurons, both $\operatorname{ER} \alpha$ and $\operatorname{ER} \beta$ could be involved in the neuroprotection of exogenously added estradiol. Contribution of ERs on the neuroprotective function of estrogen against toxicity of $\mathrm{MeHg}$ was not yet defined well.

In this study, we examined the protective action of de novo synthesized estradiol against $\mathrm{MeHg}$-induced cell death. To precisely determine estradiol synthesis in the hippocampus, rat hippocampal organotypic slice cultures were used for this study. The role of estrogen receptors in the protective effect of estradiol against $\mathrm{MeHg}$-induced neurotoxicity was also examined.

\section{Materials and Methods}

All procedures performed on animals were in accordance with the Fundamental Guidelines for Proper Conduct of Animal Experiments and Related Activities in Academic Research Institutions under the jurisdiction of the Ministry of Education, Culture, Sports, Science and Technology, Japan. The protocol was approved by the Animal Care and Use Committee of Hiroshima University, Hiroshima, Japan (The permission number is C10-32, which includes ethical approval.), and all efforts were made to minimize suffering.

\section{Rat Hippocampal Organotypic Slice Cultures}

Rat hippocampal slices were prepared from 10- to 12-day-old male Wistar rats (SLC, Shizuoka, Japan) between 10:00 am12:00 p.m. and were cultured as previously described [15,27]. Approximately $12-15$ slices of $0.3-\mathrm{mm}$ thickness were obtained from one hippocampus. Five or 6 slices from the 8 hippocampi of 4 rats were randomly placed on one of the Millicell or Omnipore membranes (Millipore, Bedford, MA) that were laid on culture plates filled with culture medium [28]. The slices were precultured at $37^{\circ} \mathrm{C}$ in a humidified incubator with $5 \% \mathrm{CO}_{2}$ for 4 days. The medium consisted of 50\% minimal essential medium, 25\% Hank's balanced salt solution (both from Sigma-Aldrich, MO, USA), and 25\% horse serum (Invitrogen, Life Technologies Corp., Carlsbad, CA, USA) supplemented with penicillin-streptomycin solution (Sigma-Aldrich), followed by a day with serum-free medium consisting of $75 \%$ minimum essential medium and $25 \%$ Hanks' balanced salt solution. After the pre-culture, $\mathrm{MeHg}$ was administered, and the slices were incubated for $24 \mathrm{~h}$. Methylmercury chloride (Tokyo Chemical Industry, Tokyo, Japan) was dissolved in Dulbecco's PBS (Sigma-Aldrich) with L-cysteine to $10 \mathrm{mM}$ each to form $\mathrm{MeHg}$-cysteine complexes (Hg:Cysteine $=1: 1)$. The $\mathrm{MeHg}$-cysteine stock solution was filtered through a membrane filter (pore size $0.45 \mu \mathrm{m}$ ) and kept at $-80^{\circ} \mathrm{C}$. The stock solution was diluted with serum-free medium just prior to use. 17 $\beta$-estradiol, 1,3,5-tris(4-hydroxyphenyl)-4propyl-1H-pyrazole (PPT; Sigma-Aldrich), 2,3-bis(4-hydroxyphenyl) propionitrile (DPN; Sigma-Aldrich), ICI 182,780 (Tocris Bioscience, Bristol, UK), or ethanol (vehicle) was administered 2 or $6 \mathrm{~h}$ before the medium change. Letrozole (extracted from Femara tablet, Novartis, Basel, Switzerland) was added $24 \mathrm{~h}$ before the addition of $\mathrm{MeHg}$.

\section{PI Uptake}

Propidium iodide (PI) is a polar compound that only enters cells with damaged membranes and becomes brightly red fluorescent after binding nucleic acids [29,30]. A concentration of $1 \mu \mathrm{M}$ PI (Sigma-Aldrich) was added to the medium $22 \mathrm{~h}$ after the administration of $\mathrm{MeHg}$. Two hours after the addition of PI, the slices were excited with a $540 \pm 25 \mathrm{~nm}$ light, and the emitted fluorescence was acquired at $605 \pm 55 \mathrm{~nm}$ on an inverted fluorescent microscope (BZ-9000, Keyence, Osaka, Japan). Fluorescence imaging was performed with Image J (http:// rsbweb.nih.gov/ij/download.html). PI uptake as a neurodegeneration index was defined as the ratio of PI fluorescence intensity after the $\mathrm{MeHg}$ treatment to putative maximal values obtained after low-temperature exposure $\left(4^{\circ} \mathrm{C}\right.$ for $\left.24 \mathrm{~h}\right)$.

\section{Quantitative RT-PCR}

The determination of mRNA levels was performed according to our previous study [15]. Briefly, the total RNA was extracted from the 20 slices after a 24-h incubation with or without $\mathrm{MeHg}$, and single-stranded cDNA was synthesized from the total RNA. Realtime PCR was performed using a LightCycler instrument (Roche Diagnostics, Basel, Switzerland) with primers described previously [15]. The amount of mRNA in MeHg-treated slices was calculated and compared to that in non-treated slices.

\section{Quantification of Estradiol}

After 4 days of pre-culture with serum-containing medium, the slices were changed to serum-free medium, and the slices were incubated $48 \mathrm{~h}$ with $\mathrm{MeHg}$, letrozole, or vehicle. To accurately measure the small amount of estradiol in the very fatty brain tissues, the steroid was extracted with an organic solvent, purified by solid column chromatography, and further purified by HPLC before analysis [31]. Estradiol in the 60 slices and the media was extracted and purified by a C18 mini-column and normal-phase HPLC as described previously [15]. The purified estradiol was quantified using an EIA kit (Cayman Chemical, Ann Arbor, MI, USA). To confirm that we were detecting chemically distinct $17 \beta$ estradiol in the EIA, some samples were also analyzed by liquid chromatography-tandem mass spectrometry (LC-MS/MS) at Asuka Pharmamedical Co. Ltd. (Kawasaki, Japan). 


\section{Statistical Analyses}

All frequencies were analyzed by one-way ANOVA for significance, and multiple comparisons using the Holm-Bonferroni method were performed to detect significant differences. $P<0.05$ was considered significant.

\section{Results}

\section{Pre-culture Period}

First, we determined the pre-culture period for the detection of tissue cell death in the rat hippocampal organotypic slice cultures. The maximum level of estradiol synthesis is typically obtained from slices after a short pre-culture time [15]. In this experiment, cell death in the cultured slices was determined by PI staining. PI uptake is a good index for damaged cells in the slices [27,32]. The fluorescence of PI staining of the slices was high at the onset of the culture, decreased to a negligible level after 5 days in culture (Fig. 1). We therefore used the slices after a 5-day pre-culture, 4 days with serum-containing medium and a day with serum-free to ensure both the high steroidogenic activity and precise analysis of tissue cell death under a low PI-staining background.

\section{The Effects of MeHg on Hippocampal Slice Cultures}

The hippocampal neuronal cell dense regions were easily detected in bright field images of the hippocampal slices (Fig. 2A). After a 5-day pre-culture, the PI fluorescence was barely detectable in the slices (Fig. 2B). After the subsequent incubation with $1 \mu \mathrm{M} \mathrm{MeHg}$, the cells in the neuronal cell dense regions were stained by PI, indicating selective vulnerability of the cells in the regions. As shown in Fig. 2C, the dentate gyrus (DG) was stained most significantly. CA1 and CA3 regions in Ammon's horn were also stained. This result is consistent with a previous observation that $\mathrm{MeHg}$-exposure during the perinatal period acutely induced cell death in the rat hippocampus, especially in the dentate gyrus [33]. The $\mathrm{MeHg}$ dose dependence of the PI uptake in the Ammon's horn and dentate gyrus is shown in Fig. 2D. After a 24-h treatment with $1 \mu \mathrm{M} \mathrm{MeHg}$, the PI uptake was approximately $50 \%$, which was suitable for the evaluation of neurotoxic effects. We used $1 \mu \mathrm{M}$ of $\mathrm{MeHg}$ for subsequent experiments.

\section{The Effects of Exogenously Added Estradiol on $\mathrm{MeHg}$ Neurotoxicity}

Incubation of slices with $10 \mu \mathrm{M}$ estradiol for $26 \mathrm{~h}$ had no effect on PI uptake in the DG, or CA1 and CA2 in Ammon's horn of the

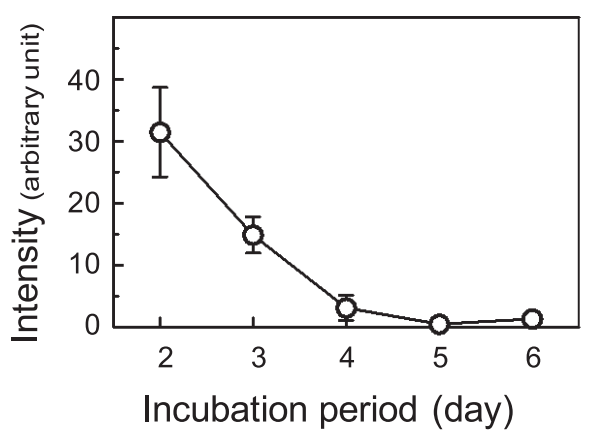

Figure 1. The PI fluorescence intensity of cultured slices. The hippocampal slices were cultured for 6 days with serum-containing medium. The intensity of PI fluorescence of whole slices is indicated. The values shown are the means of 9 slices in three different cultures, and the error bars indicate the standard error (SE).

doi:10.1371/journal.pone.0055559.g001 hippocampus (Fig. 3A). PI uptake was increased by a $24 \mathrm{~h}$ treatment with $1 \mu \mathrm{M} \mathrm{MeHg}$. Pre-treatment of the slices with $10 \mu \mathrm{M}$ estradiol $2 \mathrm{~h}$ before the addition of $1 \mu \mathrm{M} \mathrm{MeHg}$ decreased PI uptake (Fig. 3A). The neuroprotective effects of estradiol on these three regions were similar; therefore, the PI uptake was analyzed on the total neuronal cell dense region for subsequent experiments. The dose dependence of the effects of estradiol in protecting against $\mathrm{MeHg}$-induced neurotoxicity is shown in Fig. 3B. PI staining of the neuronal cell dense region in the hippocampus was completely blocked by pre-treatment with $30 \mu \mathrm{M}$ estradiol.

\section{The Effects of MeHg on mRNA and Estradiol Concentration in Hippocampal Slices}

$\mathrm{MeHg}$ has been shown to affect steroidogenic activity in the adrenal gland and gonads in various animals [10]. In mammals, brain injury induces estrogen synthesis via the rapid transcription and translation of CYP19 (P450arom) in astroglia [34]. Transcription of steroidogenic acute regulatory protein (StAR), CYP11A1 (P450scc), ER $\alpha$, and ER $\beta$ was also upregulated by injury in various brain regions [35]. Therefore, we analyzed the effect of $\mathrm{MeHg}$ on the transcript levels of these proteins and the other steroidogenic enzymes, $3 \beta$-hydroxysteroid dehydrogenase/

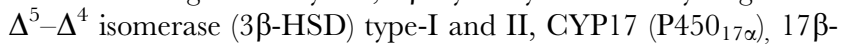
hydroxysteroid dehydrogenase (17 $\beta$-HSD) type-III and IV, and adrenal 4-binding protein/steroidogenic factor-1 (AD4BP) in the hippocampal slices by real-time RT-PCR. No significant changes were observed in mRNA levels for these proteins after incubation with or without $1 \mu \mathrm{M} \mathrm{MeHg}$ (Fig. S1).

The estradiol content of the hippocampal slices and medium was determined by EIA. The accuracy of the EIA data was crosschecked by LC-MS/MS. Estradiol contents of the slices and medium after the 48 -h incubation with or without $1 \mu \mathrm{M} \mathrm{MeHg}$ was $48.8 \pm 6.1$ and $54.9 \pm 11.2 \mathrm{fmol}$ per $\mathrm{mg}$ protein, respectively $(\mathrm{n}=3)$. Incubation with $\mathrm{MeHg}$ had no significant effect on the estradiol content of the slices and the medium.

We therefore conclude that estradiol synthesis in the slices was not affected by treatment with $1 \mu \mathrm{M} \mathrm{MeHg}$ under these experimental conditions.

\section{The Effects of an Estrogen Synthesis Inhibitor and an ER Antagonist}

Letrozole is a specific inhibitor for P450arom. The treatment of cultured hippocampal neurons and slices with letrozole induces a decrease in hippocampal estradiol synthesis [36]. The estradiol content of hippocampal slices after the 4-day pre-culture was 35.2 $\pm 4.3 \mathrm{fmol}$ per $\mathrm{mg}$ protein (Fig. 4). Then, the slices were incubated with serum-free medium for $48 \mathrm{~h}$. Estradiol content was increased to $59.8 \pm 3.0 \mathrm{fmol}$ per $\mathrm{mg}$ protein after the incubation (Fig. 4). The increment of estradiol, $25 \mathrm{fmol}$ per $\mathrm{mg}$ protein, indicated de novo synthesis in the hippocampus. After the incubation with letrozole for $48 \mathrm{~h}$, the estradiol content, $32.6 \pm 1.7 \mathrm{fmol}$ per $\mathrm{mg}$ protein, was not significantly changed from that of before incubation (Fig. 4). Letrozole treatment completely inhibited de novo estradiol synthesis in this condition.

Treatment of the slices with letrozole for $48 \mathrm{~h}$ induced no change in PI uptake, indicating that letrozole was not neurotoxic by itself (Fig. 5A). After the slices were incubated for $24 \mathrm{~h}$ with letrozole followed by $24 \mathrm{~h}$ with $1 \mu \mathrm{M} \mathrm{MeHg}$ and letrozole, the PI uptake was approximately $80 \%$, which was $30 \%$ higher than the slices treated with $\mathrm{MeHg}$ only (Fig. 5A). The increase in PI uptake was reversed by the co-addition of $3 \mu \mathrm{M}$ estradiol $2 \mathrm{~h}$ before the administration of $\mathrm{MeHg}$. These data indicate that the hippocam- 

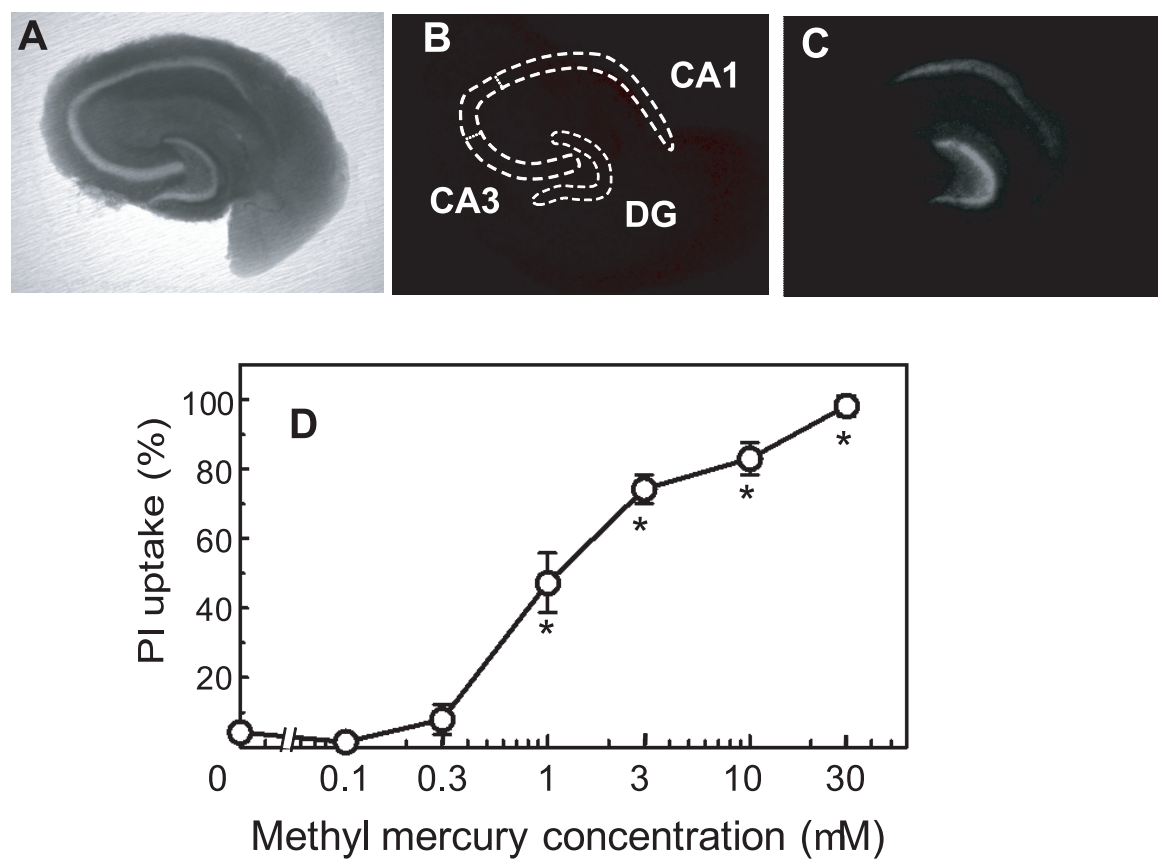

Figure 2. MeHg-induced cell death as assessed by PI uptake. Bright field (A) and PI fluorescence (B) images after 5 days of pre-culture and PI fluorescence after subsequent incubation with $1 \mu \mathrm{M}$ MeHg for $24 \mathrm{~h}(\mathrm{C})$ of the cultured hippocampal slices. Neuronal cell dense regions of dentate gyrus (DG), and CA1 and CA3 regions in Ammon's horn are traced on (A) and superimposed on (B) as dotted lines. The concentration dependency of $\mathrm{MeHg}$ on PI uptake is shown in (D). The PI uptake is expressed as a percentage of total fluorescence in the neuronal cell dense region (inside the dotted lines in B) after the slices were kept at $4^{\circ} \mathrm{C}$ for $24 \mathrm{~h}$. The values are the means of 15-21 slices in 5 different cultures, and the error bars indicate the SE. ${ }^{*}, P<0.05$, vs. MeHg-treated group, post hoc $t$-test with Holm-Bonferroni method after one-way ANOVA.

doi:10.1371/journal.pone.0055559.g002

pus-synthesized estradiol attenuated the toxic effects of $\mathrm{MeHg}$. The co-addition of $30 \mu \mathrm{M}$ estradiol blocked PI uptake almost completely, similar to the data shown in figure $3 \mathrm{~B}$, indicating no effect of letrozole on the neuroprotective effect of externally added estradiol against $\mathrm{MeHg}$.

ICI 182,780 is an antagonist both for $\mathrm{ER} \alpha$ and $\operatorname{ER} \beta$ [37]. Treatment of the slices with $100 \mu \mathrm{M}$ ICI 182,780 for $30 \mathrm{~h}$ had no effect on the PI uptake of the slices, indicating that ICI 182,780 was not neurotoxic by itself. In the presence of ICI 182,780, PI uptake after $1 \mu \mathrm{M} \mathrm{MeHg}$ treatment was increased to $82 \%$, the same level as that of the letrozole-treated slices (Fig. 5A, B). These data indicate that the protective function of de novo synthesized estradiol might be mediated by ERs in the hippocampal slices.

ICI 182,780 also blocked the protective effect of exogenously added estradiol. Pre-treatment of the slices with $3 \mu \mathrm{M}$ estradiol had no significant effect on $\mathrm{MeHg}$-induced cell death in the presence of ICI 182,780 (Fig. 5B). Interestingly, PI uptake was significantly decreased by pre-treatment with $30 \mu \mathrm{M}$ estradiol in the presence of ICI 182,780, although the protective effect was far less than in the absence of the antagonist (Figs. 3B, 5A, and 5B).

\section{The Effect of Estrogen Receptor Agonists on the Neurotoxicity of $\mathrm{MeHg}$}

PPT and DPN are specific agonists for $\mathrm{ER} \alpha$ and $\mathrm{ER} \beta$, respectively [37]. To evaluate the contribution of ERs to the protective effect, these agonists were added $2 \mathrm{~h}$ before the slices were treated with $1 \mu \mathrm{M} \mathrm{MeHg}$. PI uptake in the neuronal cell dense region was decreased by the pre-addition of these agonists in a dose-dependent manner. A significant protective effect was observed at PPT or DPN concentrations higher than $1 \mu \mathrm{M}$ (Fig. 6). These data indicate that both $\mathrm{ER} \alpha$ - and ER $\beta$-dependent pathways might mediate neuroprotection against $\mathrm{MeHg}$.

\section{Discussion}

In this study, the protective effects of hippocampal de novo synthesized estradiol against $\mathrm{MeHg}$-induced tissue cell death were demonstrated by the following results: (1) exogenously added estradiol protected rat hippocampal slices from the toxic effect of $\mathrm{MeHg}$ (Fig. 3), (2) the slices synthesized estradiol (Fig. 4), (3) de novo estradiol synthesis was not affected by $1 \mu \mathrm{M} \mathrm{MeHg}$, and (4) letrozole inhibited de novo estradiol synthesis and increased the neurotoxicity of $\mathrm{MeHg}$ (Fig. 4, 5A). Enhancement of the neurotoxic effect of $\mathrm{MeHg}$ by letrozole treatment was not caused by accumulation of estradiol precursors, because co-addition of exogenous estradiol with letrozole restored the protective effect (Fig. 5A).

The protective effect of de novo synthesized estradiol in the slices was abrogated by ICI 182,780, an ER antagonist, and was not counteracted by the addition of exogenous estradiol in the presence of the antagonist (Fig. 5B). The protective effect of estradiol was mimicked by the administration of specific agonists for $\operatorname{ER} \alpha$ or $\operatorname{ER} \beta$ (Fig. 6). The rat hippocampus possesses substantial amounts of $\mathrm{ER} \alpha$ and $\mathrm{ER} \beta$ mRNA [15]. Taken together, we concluded that the protective effect of de novo synthesized estradiol against neurotoxicity of $\mathrm{MeHg}$ was mediated by both $\mathrm{ER} \alpha$ and $\operatorname{ER} \beta$.

$\mathrm{ER} \alpha$ - and ER $\beta$-mediated neuroprotection by estradiol could be induced by classical genomic effects and/or by non-classical mechanisms $[5,8,34]$. In the classical genomic effect, ligand binding to $\mathrm{ER} \alpha$ and $\mathrm{ER} \beta$ induces dimerization of these receptors, which form either homodimers or heterodimers, to bind estrogenresponsive elements on target genes. The target genes for estradiolinduced neuroprotection have been reported to be neurotrophic factors as well as their receptors, apoptosis-related genes, and genes that modulate cellular architecture. In the non-classical 

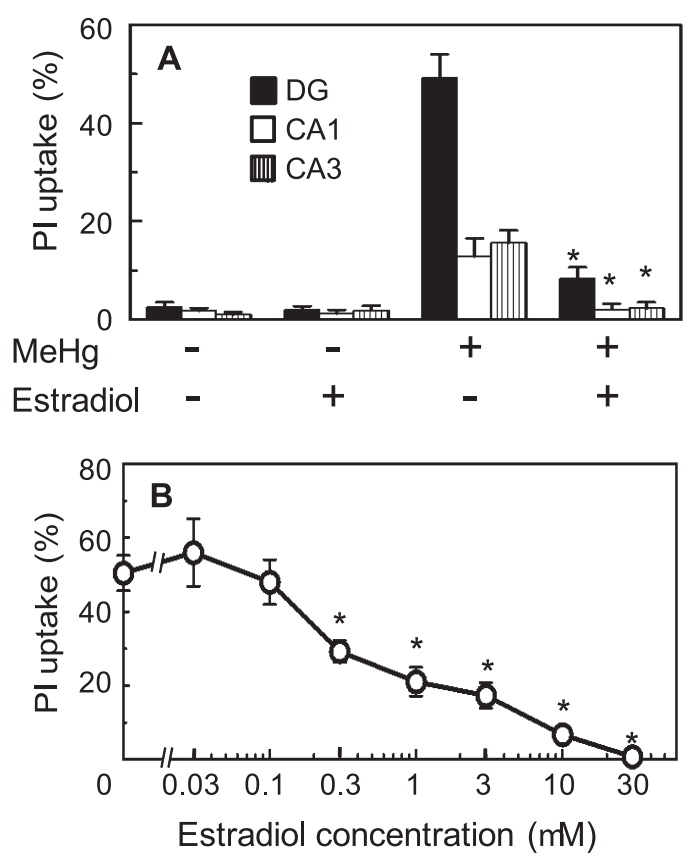

Figure 3. The effect of exogenously added estradiol on $\mathrm{MeHg}$ induced cell death. The PI uptake of the hippocampal slices was analyzed individually in the neuronal cell dense regions, dentate gyrus (DG), and CA1 and CA3 regions in Ammon's horn, which are shown in Fig. 2B (A). Slices were incubated with vehicle or $10 \mu \mathrm{M}$ estradiol for $26 \mathrm{~h}$, and/or for $24 \mathrm{~h}$ with $1 \mu \mathrm{M} \mathrm{MeHg}$. MeHg was added $2 \mathrm{~h}$ after administration of estradiol. The values are the means of 14-21 slices in 5 different cultures, and the error bars indicate the SE. ${ }^{*}, P<0.05$ vs. MeHg-treated group, post hoc $t$-test with the Holm-Bonferroni method after one-way ANOVA. The concentration dependency of the protective effect of estradiol on the PI uptake of the neuronal cell dense region of the $1 \mu \mathrm{M} \mathrm{MeHg}$-treated slice is shown in (B). The values are the mean of 14-23 slices in 4 different cultures, and the error bars indicate the SE. *, $P<0.05$ vs. MeHg-treated group, post hoc $t$-test with Holm-Bonferroni method after one-way ANOVA.

doi:10.1371/journal.pone.0055559.g003

mechanisms of ERs, various signal transduction pathways are activated by estradiol, including intracellular $\mathrm{Ca}^{2+}$, extracellularsignal regulated kinases 1 and 2 (ERK1/2), and G-protein-coupled receptors, etc. The neurotoxicity of $\mathrm{MeHg}$ may be mediated by molecular mechanisms of a variety of cellular functions, including intracellular $\mathrm{Ca}^{2+}$ and glutathione homeostasis, maintenance of the mitochondrial membrane potential, assembly and disassembly of microtubules, and activity of the $\mathrm{Na}^{+} / \mathrm{K}^{+}$pump [38]. $\mathrm{MeHg}$ exposure generates reactive oxygen species and radicals in neurons, which may induce cell damage [39,40,41]. The precise molecular mechanism of the neuroprotective effect of estradiol in rat hippocampal slices is currently under investigation in our laboratory.

Although the protective effects of de novo synthesized and lower concentrations of exogenous estradiol were mediated by the ERs, $30 \mu \mathrm{M}$ exogenous estradiol partly protected hippocampal cells from the toxic effects of $\mathrm{MeHg}$ in the presence of ICI 182,780 (Fig. 5A). This high concentration of estradiol might protect against cell death without activation of the estrogen receptor system. Estradiol itself is a neuroprotective antioxidant at concentrations higher than a few micromolar [5,8]. Indeed, the neurotoxic effect of $\mathrm{MeHg}$ on cultured cerebellar granule cells was abrogated by the antioxidant function of $10 \mu \mathrm{M}$ estradiol [11]. On the other hand, multiple molecular mechanisms for receptor-

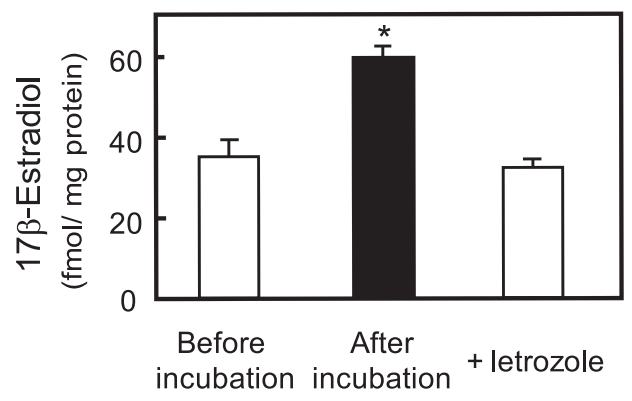

Figure 4. The inhibition of estradiol synthesis by letrozole. Estradiol was extracted from slices and medium before and after a 48-h incubation with or without letrozole. Letrozole was extracted from Femara tablets. The levels of extracted $17 \beta$-estradiol were quantified as described in the Materials and Methods section. The values are the means of 3 separate experiments, and the error bars indicate the SE. *, $P<0.05$, vs. non-treated group, $t$-test.

doi:10.1371/journal.pone.0055559.g004

independent neuroprotection by estradiol have been proposed. The indirect genomic mechanism requires the rapid activation of mitogen-activated protein kinases and Akt signaling pathways and estrogen-induced activation of a transcription factor cAMP-
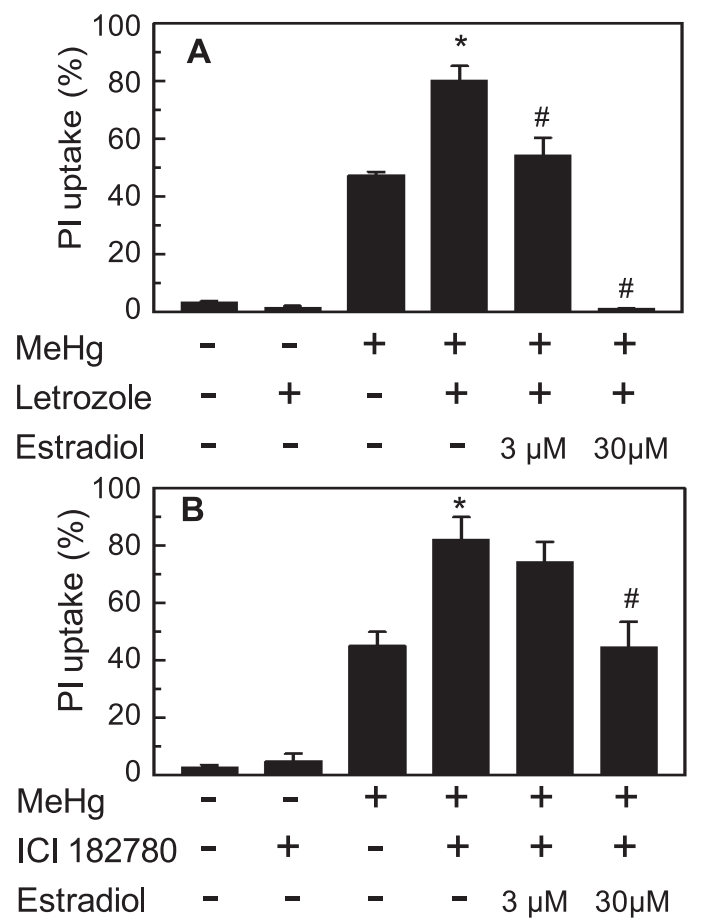

Figure 5. The effects of letrozole and ICI on MeHg-induced cell death. Levels of PI uptake in the neuronal cell dense region of the slices after a 48-h treatment with vehicle or letrozole and/or 24-h treatment with $1 \mu \mathrm{M} \mathrm{MeHg}$ are shown in A. Levels of PI uptake after a 30-h treatment with vehicle or $100 \mu \mathrm{M} \mathrm{ICl} \mathrm{182,780} \mathrm{and/or} \mathrm{24-h}$ treatment with $1 \mu \mathrm{M} \mathrm{MeHg}$ are shown in $\mathrm{B}$. MeHg was added 24 or $6 \mathrm{~h}$ after the administration of Letrozole or ICI 182,780, respectively. Some wells were given the indicated concentrations of estradiol $2 \mathrm{~h}$ before the addition of MeHg. The values are the means of 16-21 slices in 5 different cultures or 13-16 slices in 4 different cultures for A or B, respectively, and the error bars indicate the SE. ${ }^{*}, P<0.05$, vs. the MeHgtreated group, and \#, $P<0.05$, vs. $\mathrm{MeHg}$ and letrozole- or ICl-treated group, post hoc $t$-test with the Holm-Bonferroni method after one-way ANOVA.

doi:10.1371/journal.pone.0055559.g005 

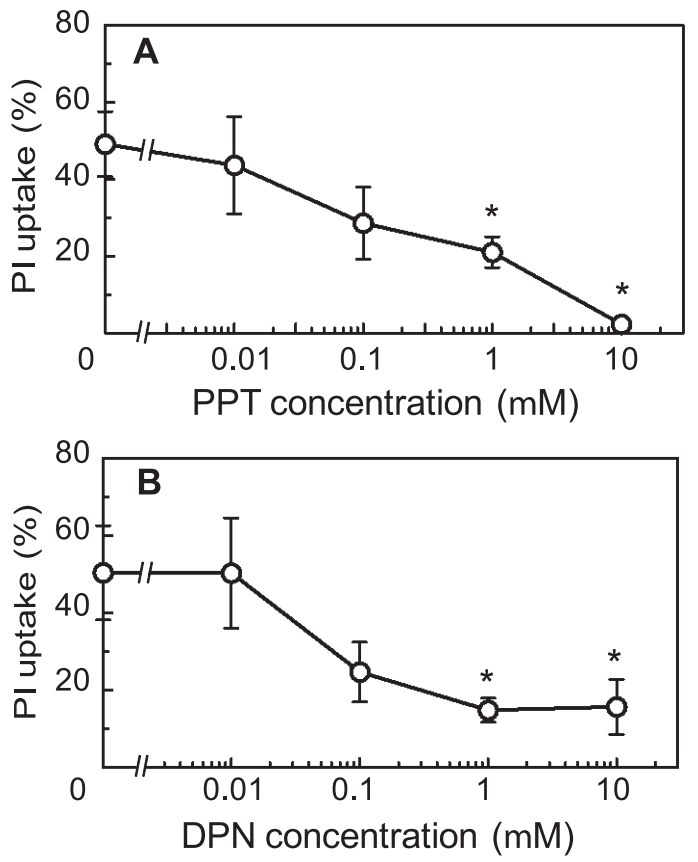

Figure 6. The effects of estrogen receptor agonists on $\mathrm{MeHg}$ induced cell death. The PI uptake of neuronal cell dense region of the slices was determined after a 24-h treatment with $1 \mu \mathrm{M} \mathrm{MeHg}$ in the presence of various concentrations of PPT (A) or DPN (B). These agonists were administered $2 \mathrm{~h}$ before the addition of $\mathrm{MeHg}$. The values are the means of 16-22 slices in four different cultures, and the error bars indicate the SE. ${ }^{*}, P<0.05$, vs. MeHg-treated group, post hoc $t$-test with Holm-Bonferroni method after one-way ANOVA.

doi:10.1371/journal.pone.0055559.g006

responsive element binding protein [5,8]. The receptor-independent mechanisms of neuroprotection by estradiol are also under examination in our laboratory.

In this in vitro study, estradiol synthesis in the hippocampal slices was monitored by determination of actual estradiol contents in the slice to evaluate the significance of de novo estradiol synthesis on the neuronal self-protection. Our results are consistent with an in vivo study; intracerebral administration of fadrozole, another P450arom inhibitor, enhanced kainic acid-induced neurodegeneration in the hippocampus of the male rat, although de novo estradiol synthesis was not quantified in the in vivo brain [42]. Both their study and ours are, however, lacking in information about the source of the de novo estradiol synthesis. In our in vitro experiment, slices were cultured in serum-free medium, which did not contain detectable steroids. We have previously reported that stimulation of de novo estradiol synthesis in the slices by 9-cisretinoic acid is concomitant with increases in de novo testosterone synthesis and the mRNA level of $\mathrm{P} 450_{17 \alpha}$, indicating that the precursors of these steroids are located upstream of $\mathrm{P} 450_{17 \alpha}$ activity, such as pregnenolone, progesterone, and/or cholesterol [15]. In in vivo hippocampus, estradiol might be synthesized from precursors from both inside and outside the brain. Plasma

\section{References}

1. McEwen B (2002) Estrogen actions throughout the brain. Recent Prog Horm Res 57: 357-384.

2. Bourque M, Dluzen DE, Di Paolo T (2009) Neuroprotective actions of sex steroids in Parkinson's disease. Front Neuroendocrinol 30: 142-157.

3. Pike CJ, Carroll JC, Rosario ER, Barron AM (2009) Protective actions of sex steroid hormones in Alzheimer's disease. Front Neuroendocrinol 30: 239-258. testosterone has high blood-brain barrier permeability and may be a substrate for P450arom [43]. Decrease in plasma testosterone by castration, however, only affected hippocampal estradiol contents very slightly. Estradiol content in castrated rat hippocampus was $83 \%$ of that in sham-operated rats, although plasma testosterone concentration was decreased to one-seventieth [31]. The in vivo hippocampus might synthesize estradiol also from substrates other than plasma testosterone, such as pregnenolone, progesterone and/or cholesterol.

Brain estradiol can be derived from peripheral steroidogenic organs via the blood stream, in addition to de novo synthesis. Plasma estradiol may distribute through the entire brain because estradiol has high blood-brain barrier permeability [43]. The de novo estradiol synthesis is, however, limited to discrete regions and varies by developmental stage and animal species [12]. The selfprotective effect of de novo synthesized estradiol may be regionspecific in the brain and vary by developmental stage and animal species. Indeed, the estradiol content and the mRNA level for P450arom in the cerebral cortex was about one-half and one-fifth, respectively, of those in the hippocampus of 10-day-old rats (unpublished result). This result is consistent with the observation that the neuronal damage in the cerebral cortex was higher than that in the hippocampus of 15-day-old rats after administration of $\mathrm{MeHg}$ [44]. In the mouse brain, $\mathrm{MeHg}$ induced neuropathological changes in the cerebral cortex but not in the hippocampus, despite a similar accumulation of mercury [45]. The pathological changes observed in Minamata disease occur predominantly in specific areas, including the calcarine region, the postcentral and precentral gyri, and the temporal transverse gyrus [24]. Mercury was, however, detected over a wide area of the brain including the thalamus, pallidum and caudate nucleus with Minamata disease following a 26 year clinical course after the first severe attack in 1956 [46]. Although de novo estrogen synthesis in local areas of human brain is not yet well analyzed, mRNA for P450arom was lower in cerebellum than thalamus [47]. To understand the molecular mechanism for the region specific lesions in different brain areas, the self-protective effect of de novo synthesized estradiol is worth considering as one of the possible factors.

\section{Supporting Information}

Figure S1 The effect of MeHg on mRNA levels in the slices. The slices were incubated with or without $1 \mu \mathrm{M} \mathrm{MeHg}$ for $24 \mathrm{~h}$. The amounts of mRNA were determined by real-time RTPCR as described in the Materials and Methods section. The amounts of mRNA in MeHg-treated slices were given as the values relative to that in the non-treated slices. The values are the means of 3 separate experiments, and the error bars indicate the SE. (EPS)

\section{Author Contributions}

Conceived and designed the experiments: TY MY. Performed the experiments: YI S. Komatsu EM MO TM. Analyzed the data: TY. Contributed reagents/materials/analysis tools: AI S. Kawato. Wrote the paper: TY MY.

4. Suzuki S, Brown CM, Wise PM (2009) Neuroprotective effects of estrogens following ischemic stroke. Front Neuroendocrinol 30: 201-211.

5. Behl C (2002) Oestrogen as a neuroprotective hormone. Nat Rev Neurosci 3: 433-442.

6. Haynes LE, Lendon CL, Barber DJ, Mitchell IJ (2003) 17 Beta-oestradiol attenuates dexamethasone-induced lethal and sublethal neuronal damage in the striatum and hippocampus. Neuroscience 120: 799-806. 
7. Lee ES, Yin Z, Milatovic D, Jiang H, Aschner M (2009) Estrogen and tamoxifen protect against Mn-induced toxicity in rat cortical primary cultures of neurons and astrocytes. Toxicol Sci 110: 156-167.

8. Singh M, Dykens JA, Simpkins JW (2006) Novel mechanisms for estrogeninduced neuroprotection. Exp Biol Med (Maywood) 231: 514-521.

9. Yi KD, Covey DF, Simpkins JW (2009) Mechanism of okadaic acid-induced neuronal death and the effect of estrogens. J Neurochem 108: 732-740.

10. Tan SW, Meiller JC, Mahaffey KR (2009) The endocrine effects of mercury in humans and wildlife. Crit Rev Toxicol 39: 228-269.

11. Dare E, Gotz ME, Zhivotovsky B, Manzo L, Ceccatelli S (2000) Antioxidants J811 and 17beta-estradiol protect cerebellar granule cells from methylmercuryinduced apoptotic cell death. J Neurosci Res 62: 557-565.

12. Baulieu EE (1997) Neurosteroids: of the nervous system, by the nervous system, for the nervous system. Recent Prog Horm Res 52: 1-32.

13. Konkle AT, McCarthy MM (2011) Developmental time course of estradiol, testosterone, and dihydrotestosterone levels in discrete regions of male and female rat brain. Endocrinology 152: 223-235.

14. Kretz O, Fester L, Wehrenberg U, Zhou L, Brauckmann S, et al. (2004) Hippocampal synapses depend on hippocampal estrogen synthesis. J Neurosci 24: 5913-5921.

15. Munetsuna E, Hojo Y, Hattori M, Ishii H, Kawato S, et al. (2009) Retinoic acid stimulates 17 beta-estradiol and testosterone synthesis in rat hippocampal slice cultures. Endocrinology 150: 4260-4269.

16. Fester L, Prange-Kiel J, Jarry H, Rune GM (2011) Estrogen synthesis in the hippocampus. Cell Tissue Res 345: 285-294.

17. Hojo Y, Hattori TA, Enami T, Furukawa A, Suzuki K, et al. (2004) Adult male rat hippocampus synthesizes estradiol from pregnenolone by cytochromes P45017alpha and P450 aromatase localized in neurons. Proc Natl Acad Sci U S A 101: 865-870.

18. Aschner M, Syversen T (2005) Methylmercury: recent advances in the understanding of its neurotoxicity. Ther Drug Monit 27: 278-283.

19. Clarkson TW (1997) The toxicology of mercury. Crit Rev Clin Lab Sci 34: 369 403.

20. Bakir F, Damluji SF, Amin-Zaki L, Murtadha M, Khalidi A, et al. (1973) Methylmercury poisoning in Iraq. Science 181: 230-241.

21. Eto K (2000) Minamata disease. Neuropathology 20 Suppl: S14-19.

22. Takeuchi T (1982) Pathology of Minamata disease. With special reference to its pathogenesis. Acta Pathol Jpn 32 Suppl 1: 73-99.

23. Okabe M, Takeuchi T (1980) Distribution and fate of mercury in tissue of human organs in Minamata disease. Neurotoxicology 1: 607-624.

24. Eto K (1997) Pathology of Minamata disease. Toxicol Pathol 25: 614-623.

25. Malagutti KS, da Silva AP, Braga HC, Mitozo PA, Soares Dos Santos AR, et al. (2009) 17beta-estradiol decreases methylmercury-induced neurotoxicity in male mice. Environ Toxicol Pharmacol 27: 293-297.

26. Zhao L, Wu TW, Brinton RD (2004) Estrogen receptor subtypes alpha and beta contribute to neuroprotection and increased Bcl-2 expression in primary hippocampal neurons. Brain Res 1010: 22-34.

27. Ishihara Y, Kawami T, Ishida A, Yamazaki T (2012) Tributyltin induces oxidative stress and neuronal injury by inhibiting glutathione $\mathrm{S}$-transferase in rat organotypic hippocampal slice cultures. Neurochem Int 60: 782-790.

28. Koyama R, Muramatsu R, Sasaki T, Kimura R, Ueyama C, et al. (2007) A lowcost method for brain slice cultures. J Pharmacol Sci 104: 191-194.
29. Macklis JD, Madison RD (1990) Progressive incorporation of propidium iodide in cultured mouse neurons correlates with declining electrophysiological status: a fluorescence scale of membrane integrity. J Neurosci Methods 31: 43-46.

30. Vitale M, Zamai L, Mazzotti G, Cataldi A, Falcieri E (1993) Differential kinetics of propidium iodide uptake in apoptotic and necrotic thymocytes. Histochemistry 100: 223-229.

31. Hojo Y, Higo S, Ishii H, Ooishi Y, Mukai H, et al. (2009) Comparison between hippocampus-synthesized and circulation-derived sex steroids in the hippocampus. Endocrinology 150: 5106-5112.

32. Zimmer J, Kristensen BW, Jakobsen B, Noraberg J (2000) Excitatory amino acid neurotoxicity and modulation of glutamate receptor expression in organotypic brain slice cultures. Amino Acids 19: 7-21.

33. Falluel-Morel A, Sokolowski K, Sisti HM, Zhou X, Shors TJ, et al. (2007) Developmental mercury exposure elicits acute hippocampal cell death, reductions in neurogenesis, and severe learning deficits during puberty. J Neurochem 103: 1968-1981.

34. Saldanha CJ, Duncan KA, Walters BJ (2009) Neuroprotective actions of brain aromatase. Front Neuroendocrinol 30: 106-118.

35. Garcia-Ovejero D, Azcoitia I, Doncarlos LL, Melcangi RC, Garcia-Segura LM (2005) Glia-neuron crosstalk in the neuroprotective mechanisms of sex steroid hormones. Brain Res Brain Res Rev 48: 273-286.

36. Rune GM, Lohse C, Prange-Kiel J, Fester L, Frotscher M (2006) Synaptic plasticity in the hippocampus: effects of estrogen from the gonads or hippocampus? Neurochem Res 31: 145-155.

37. Dahlman-Wright K, Cavailles V, Fuqua SA, Jordan VC, Katzenellenbogen JA, et al. (2006) International Union of Pharmacology. LXIV. Estrogen receptors. Pharmacol Rev 58: 773-781.

38. Nagashima K (1997) A review of experimental methylmercury toxicity in rats: neuropathology and evidence for apoptosis. Toxicol Pathol 25: 624-631.

39. Sarafian TA, Magallanes JA, Shau H, Tashkin D, Roth MD (1999) Oxidative stress produced by marijuana smoke. An adverse effect enhanced by cannabinoids. Am J Respir Cell Mol Biol 20: 1286-1293.

40. Yee S, Choi BH (1996) Oxidative stress in neurotoxic effects of methylmercury poisoning. Neurotoxicology 17: 17-26.

41. Ceccatelli S, Dare E, Moors M (2010) Methylmercury-induced neurotoxicity and apoptosis. Chem Biol Interact 188: 301-308.

42. Garcia-Segura LM, Veiga S, Sierra A, Melcangi RC, Azcoitia I (2003) Aromatase: a neuroprotective enzyme. Prog Neurobiol 71: 31-41.

43. Pardridge WM, Mietus LJ (1979) Transport of steroid hormones through the rat blood-brain barrier. Primary role of albumin-bound hormone. J Clin Invest 64: $145-154$.

44. Wakabayashi K, Kakita A, Sakamoto M, Su M, Iwanaga K, et al. (1995) Variability of brain lesions in rats administered methylmercury at various postnatal development phases. Brain Res 705: 267-272.

45. Fujimura M, Usuki F, Sawada M, Takashima A (2009) Methylmercury induces neuropathological changes with tau hyperphosphorylation mainly through the activation of the c-jun-N-terminal kinase pathway in the cerebral cortex, but not in the hippocampus of the mouse brain. Neurotoxicology 30: 1000-1007.

46. Takeuchi T, Eto K, Tokunaga H (1989) Mercury level and histochemical distribution in a human brain with Minamata disease following a long-term clinical course of twenty-six years. Neurotoxicology 10: 651-657.

47. Sasano H, Takashashi K, Satoh F, Nagura H, Harada N (1998) Aromatase in the human central nervous system. Clin Endocrinol (Oxf) 48: 325-329. 